\title{
Microvascular contrast enhancement in optical coherence tomography using microbubbles
}

Homa Assadi

Valentin Demidov

Raffi Karshafian

Alexandre Douplik

I. Alex Vitkin 


\title{
Microvascular contrast enhancement in optical coherence tomography using microbubbles
}

\author{
Homa Assadi, ${ }^{a, t, *}$ Valentin Demidov, ${ }^{b, \dagger}$ Raffi Karshafian, ${ }^{a, c}$ Alexandre Douplik, ${ }^{a, c}$ and I. Alex Vitkin ${ }^{\text {b,d,e }}$ \\ ${ }^{a}$ Ryerson University, Department of Physics, 350 Victoria Street, Toronto, Ontario M5B 2K3, Canada \\ bUniversity of Toronto, Department of Medical Biophysics, Toronto Medical Discovery Tower, MaRS Centre, 101 College Street, Room 15-701, \\ Toronto, Ontario M5G 1L7, Canada \\ 'St. Michael Hospital, Keenan Research Centre of the LKS Knowledge Institute, 209 Victoria Street, Toronto M5B 1W8, Canada \\ dUniversity Health Network, Princess Margaret Cancer Centre, 610 University Avenue, Toronto, Ontario M5G 2M9, Canada \\ eUniversity of Toronto, Department of Radiation Oncology, 150 College Street, FitzGerald Building, Room 106, Toronto, Ontario M5S 3E2, Canada
}

\begin{abstract}
Gas microbubbles (MBs) are investigated as intravascular optical coherence tomography (OCT) contrast agents. Agar + intralipid scattering tissue phantoms with two embedded microtubes were fabricated to model vascular blood flow. One was filled with human blood, and the other with a mixture of human blood + MB. Swept-source structural and speckle variance (sv) OCT images, as well as speckle decorrelation times, were evaluated under both no-flow and varying flow conditions. Faster decorrelation times and higher structural and sVOCT image contrasts were detected in the presence of MB in all experiments. The effects were largest in the svOCT imaging mode, and uniformly diminished with increasing flow velocity. These findings suggest the feasibility of utilizing MB for tissue hemodynamic investigations and for microvasculature contrast enhancement in OCT angiography. ( 2016 Society of Photo-Optical Instrumentation Engineers (SPIE) [DOI: 10.1117/1.JBO.21.7 .076014]
\end{abstract}

Keywords: microbubbles; contrast agents; optical coherence tomography; microcirculation; contrast enhancement; speckle variance. Paper 150750R received Nov. 6, 2015; accepted for publication Jul. 11, 2016; published online Jul. 28, 2016.

\section{Introduction}

Various contrast agents have been suggested to enhance tissue microvasculature in optical coherence tomography (OCT), ${ }^{1}$ including gold nanoparticles, ${ }^{2}$ nanorods, ${ }^{3}$ microspheres, ${ }^{4}$ and nanoshells. ${ }^{5}$ Gold nanoparticles enhanced the microvascular contrast in vivo in an oral dysplasia hamster cheek pouch model by as much as $150 \% .{ }^{6}$ Microspheres with liquid-filled cores and scattering particles within their shell have also shown significant contrast improvement, and deployment of these contrast media has been demonstrated for in-vivo OCT. ${ }^{7}$ However, while initially in the blood stream, these agents leak from the vessels to the surrounding tissues which decreases the contrast, and their accumulation (often in liver) may cause a number of side effects. ${ }^{8}$ In light of these drawbacks, microbubbles [MBs; currently Food and Drug Administration (FDA) approved intravascular contrast agents in diagnostic ultrasound $\left.{ }^{9}\right]$ may have a role to play as OCT contrast agents. ${ }^{10,11}$

MBs are micrometer sized shell-encapsulated gas-core spheres. They are considered intravascular agents, i.e., following intravenous injection, they remain within the blood compartment and do not extravasate. Their pharmacokinetics suggest a lifetime of $\sim 10$ to $40 \mathrm{~min}$ following injection, depending on their shell composition and gas type. ${ }^{12}$ In addition to diagnostic/ imaging applications, MBs (in combination with therapeutic ultrasound) are being investigated to enhance chemotherapy, ${ }^{13,14}$ radiotherapy, ${ }^{15}$ and thermal therapy with gold nanoparticles. ${ }^{16}$

Speckle variance (sv) OCT has been actively pursued as an attractive alternative for three-dimensional (3-D) visualization

*Address all correspondence to: Homa Assadi, E-mail: homa.assadi@ryerson ca

TThese authors contributed equally to this work. of the microvascular network, utilizing temporal variation of the scattering (speckle) pattern between frames as the contrast mechanism. ${ }^{17,18}$ In addition to the high resolution svOCT imaging of microvasculature, it has potential to provide new insight into the dynamics of vascular remodeling. ${ }^{19-21}$

In our previous phantom study, ${ }^{11}$ we demonstrated that MBs can be used as an optical contrast agent, yielding increased scattering $(1.4 \times$ at $300 \mathrm{~nm}$ and $6 \times$ at $850 \mathrm{~nm})$. Similarly, Barton et al. ${ }^{10}$ have shown that MBs enhanced the backscattered OCT signal in model scattering media. Initial in-vivo work also yielded enhanced visualization of blood vessels with lumen diameters less than a few hundred micrometers. ${ }^{10}$ However, a quantitative assessment of MB contrast enhancement under noflow and flowing blood conditions in a well-controlled model system with known parameters is lacking, and is addressed in the current study.

\section{Materials and Methods}

\subsection{Tissue Phantoms}

To model biological tissue, 10 phantoms were made by heating $1 \%$ TCBS agar (Sigma-Aldrich, Missouri) and 2\% intralipid diluted from 20\% stock (Sigma-Aldrich, St. Louis, Missouri) in water. Intralipid mimics the scattering properties of biological tissue, ${ }^{22,23}$ and agar solidifies the resultant suspension upon cooling. The heated mixture was cooled in chambers containing two microtubes to simulate blood vessels. Five prepared phantom chambers contained microtubes with internal diameters of $600 \mu \mathrm{m}$, and five more with internal diameters of $300 \mu \mathrm{m}$. A double-syringe high-precision pump (Model NE-4002X, New Era Pump Systems, Farmingdale, New York) was used to

$1083-3668 / 2016 / \$ 25.00$ @ 2016 SPIE 
fill the two microtubes simultaneously; OCT measurements under both no-flow and flow conditions were then possible. The average blood flow velocities in both microtubes were 0 , $0.5,1,2,3,5,8$, and $12 \mathrm{~mm} / \mathrm{s}$ corresponding to venules, capillaries, and arterioles. ${ }^{24-26}$ One syringe was filled with blood, the other with the mixture of blood and MBs. Rotating magnets inside both syringes were used to avoid red blood cells (RBC) settling and MBs floating during all experiments.

\subsection{Human Blood}

Ethics approval was granted by the Research Ethics Boards of Ryerson University and the Canadian Blood Services. All experiments were conducted using whole blood donated by healthy volunteers recruited by the Canadian Blood Services' Network Center for Applied Development (Vancouver, BC, Canada). The whole blood was 1-week old and contained $14 \%$ citrate phosphate dextrose anticoagulant.

\subsection{Microbubbles}

Commercially available Definity ${ }^{\circledR}$ microbubbles (perflutren lipid microspheres, Lantheus Medical Imaging, Billerica, Massachusetts), FDA approved for diagnostic ultrasound contrast, were used in this study. The mean diameter of MBs was $1.2 \pm 0.5 \mu \mathrm{m}$. A $2.5 \%$ volume fraction of a stock suspension (average $\sim 1.2 \times 10^{10} \mathrm{MBs} / \mathrm{mL}$ ) was injected into the blood microtube. The resultant $\mathrm{MB}$ concentration was comparable to that used in ex-vivo ultrasound tests. ${ }^{27}$

\subsection{Optical Coherence Tomography System}

A home-built and previously described swept source OCT system $^{28}$ was used in this study. Briefly, a $36 \mathrm{kHz}$ short cavity laser source with a polygon-based tuneable filter and wavelength centered at $1310 \mathrm{~nm}$ had a sweep range of $110 \mathrm{~nm}$ and an average output power of $40 \mathrm{~mW}$. The system contained a 50/50 Mach-Zehnder interferometer and an imaging probe mounted on three-axis micropositioning stage in the sample arm. A dual-balanced photo detector was used in the output arm of the interferometer for DC signal component removal. A fiber Bragg grating provided the A-scan trigger at the beginning of each laser sweep. An OCT signal recalibration was performed with a standard Mach-Zehnder recalibration interferometer to generate linear k-space data from the raw data sampled with a $125 \mathrm{MS} / \mathrm{s}$ data acquisition card. After the data were equally

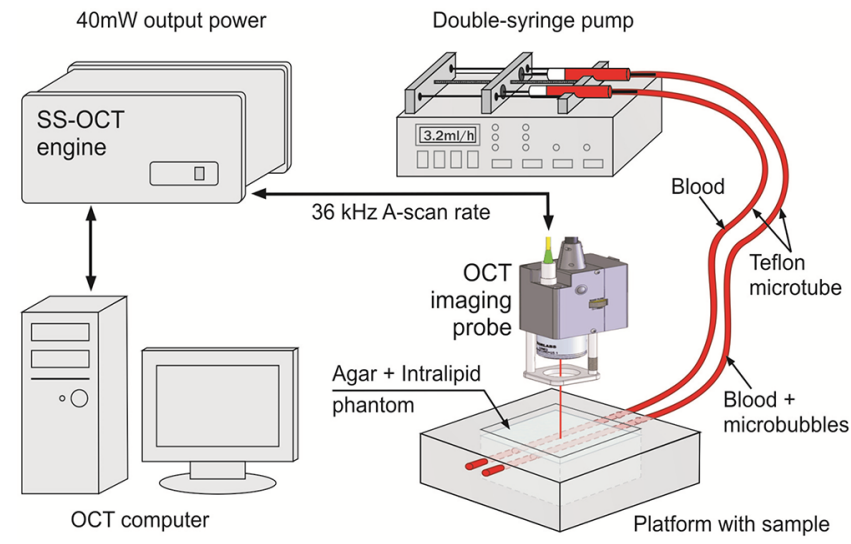

Fig. 1 Experimental setup. spaced in k-space, it was inversely Fourier transformed for further analysis. The axial resolution (in air) of the system was $8 \mu \mathrm{m}$. The experimental setup is shown in Fig. 1.

\subsection{Data Acquisition and Processing}

OCT imaging was performed for both no-flow and flow conditions for blood (left microtube on subsequent images) and blood $+\mathrm{MB}$ mixture (right microtube). Data were recorded using Thorlabs software (Thorlabs Inc., Newton, New Jersey) in one-dimensional (1-D), two-dimensional (2-D), and 3-D modes.

In 1-D, A-scans were acquired from both microtubes to quantify MB-induced differences in OCT signal intensities. The autocorrelation function (ACF) was calculated from 18 pixels along the chosen A-line (shown in Fig. 2 as a vertical white dashed line) within the region of interest (ROI) of the corresponding microtube. The average of speckle decorrelation times $(1 / e$ level) of these 18 pixels is displayed in Fig. 3. Autocorrelation was considered as the correlation between intensity values $I_{i}$ of the same pixel at times $t_{i}$ and $t_{i+\tau}$ with increasing time delay $\tau$ :

$\mathrm{ACF}_{i}=\frac{\sum_{i=1}^{N-\tau}\left(I_{i}-\bar{I}\right)\left(I_{i+\tau}-\bar{I}\right)}{\sum_{i=1}^{N}\left(I_{i}-\bar{I}\right)^{2}}$.

For each phantom, A-scans were recorded continuously for $8 \mathrm{~s}$ with the optical beam parked at the center of the chosen microtube (with blood or blood $+\mathrm{MB}$ ) for all flow conditions. Each

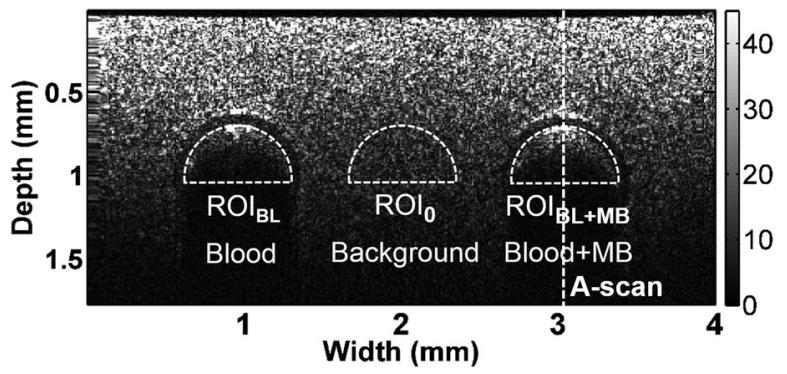

Fig. 2 Typical structural B-scan of the phantom. The left microtube was filled with blood, and the right with blood + MBs $[2.5 \%$ volume fraction of a stock suspension (average $\sim 1.2 \times 10^{10} \mathrm{MBs} / \mathrm{mL}$ ) were injected into the blood microtube]. ROls of blood and blood + MB mixture are labeled with half-circles at the top circular section of microtube lumens. Background ROI was chosen at the same depth as shown. The vertical dashed line indicates the location of the acquired A-scans for ACF calculation (Fig. 3).

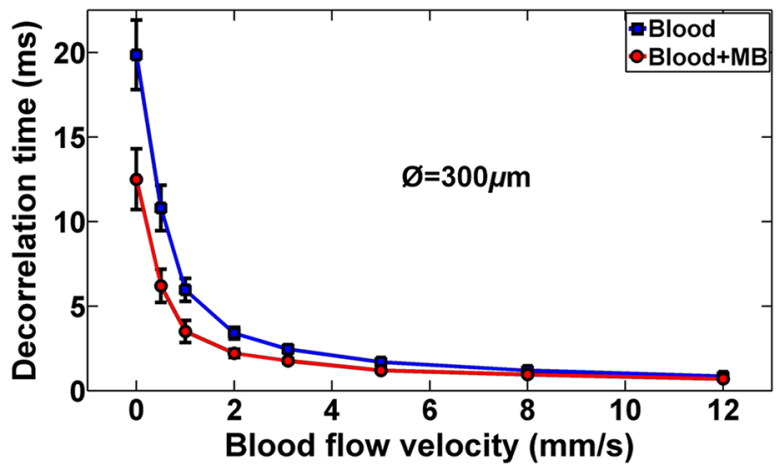

Fig. 3 Decorrelation time plots for blood and blood $+\mathrm{MB}$ mixture under eight flow conditions in $300-\mu \mathrm{m}$ tube. 
resultant record of 288,000 A-scans was divided into 240 intervals of $N=1200$ A-scans ( $33 \mathrm{~ms}$ acquisition time). ACFs were computed for each interval and then averaged.

2-D images (B-scans) of each phantom were acquired with $4 \mathrm{~mm}$ of lateral scanning. MATLAB software (MATLAB, Mathworks, Natick, Massachusetts) was used for data processing. Images of both blood and blood $+\mathrm{MB}$ microtubes were used to quantify the difference in resultant phantom contrasts. Since the majority of the signal from the bottom of the microtube was low due to high scattering of blood, the ROI was chosen as the top-half circular section of the microtube lumen (see Fig. 2). The averaged ROI intensity was used to quantify the contrast via

$C_{\mathrm{W}}=\frac{I-I_{0}}{I_{0}}$

where $I$ is the average intensity within ROI and $I_{0}$ is the average intensity of the background $\mathrm{ROI}_{0}$. The background $\mathrm{ROI}_{0}$ was chosen to be in the middle of the phantom between the microtubes, at the same depth (Fig. 2).

3-D OCT images were acquired in sv mode in order to obtain sv average intensity projections for $3 \times 1 \times 1 \mathrm{~mm}^{3}$ data volume as previously described, ${ }^{28}$ with the number of sequential same location B-scans equal to 8 , and $25 \mathrm{~ms}$ step size in slow-axis $(Y)$ direction. svOCT calculates the interframe intensity variance of structural images, where contrast is based on the difference in time-varying properties. Specifically,

$\mathrm{SV}_{j k}=\frac{1}{N} \sum_{i=1}^{N}\left(I_{i j k}-\overline{I_{j k}}\right)^{2}$

where $N=8$ is the number of B-scans acquired from the same $i$-location, $I_{i j k}$ is the intensity of the pixel of the $i$ 'th B-scan, $j$ is the axial pixel, $k$ is the lateral pixel, and $\overline{I_{j k}}$ is the mean intensity of that pixel over eight sequential B-scans. Statistical analysis (two-tailed test) was performed using SPSS statistics software, using results from the five phantoms for any given experimental point. Results with $p<0.05$ were considered statistically significant.

\section{Results and Discussion}

\subsection{One-Dimensional Decorrelation Analysis}

The ACFs of OCT signals with and without MBs at no-flow and varying flow conditions are plotted in Fig. 3. The results from the 300- $\mu$ m-diameter microtube are shown; those from the larger microtube show similar trends with slightly higher values at low flows. As expected, the decorrelation time decreases with blood
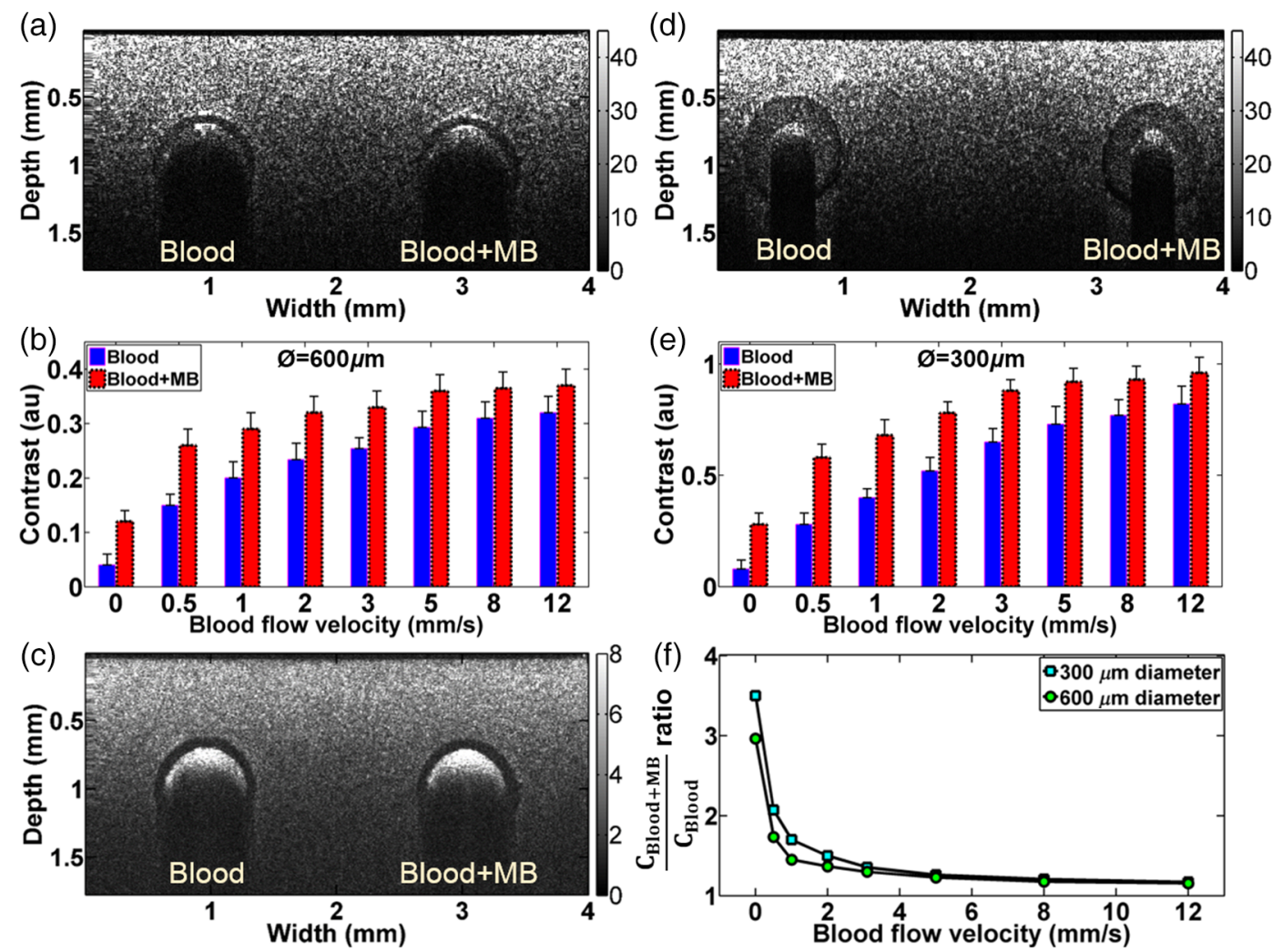

Fig. 4 Structural images, corresponding contrast of blood and blood + MB mixture and ratios of blood + MB to blood contrasts under eight flow conditions for 600- and 300- $\mu \mathrm{m}$ microtubes. (a) Structural image of the phantom with $600-\mu \mathrm{m}$ microtubes at $0.5 \mathrm{~mm} / \mathrm{s}$ blood flow velocity; (b) structural image contrast for $600-\mu \mathrm{m}$ microtubes; (c) standard deviation image calculated from eight sequential structural images at $0.5 \mathrm{~mm} / \mathrm{s}$ blood flow velocity; (d) structural image of the phantom with $300-\mu \mathrm{m}$ microtubes at $0.5 \mathrm{~mm} / \mathrm{s}$ blood flow velocity; (e) structural image contrast for 300- $\mu \mathrm{m}$ microtubes. Error bars in (b) and (e) were evaluated from data at 200 different locations (B-scans) within each phantom. Note the different vertical scales in (b) and (e), see text for explanation; (f) ratio of blood $+M B / b l o o d$ structural contrasts for both microtube diameters. 
flow velocity. The decorrelation times were typically $\sim 15 \mathrm{~ms}$ at no-flow condition, decreasing to $\sim 1 \mathrm{~ms}$ for velocities $>5 \mathrm{~mm} / \mathrm{s}$. As seen, the presence of MB causes faster decorrelation, and this differential effect diminishes with increasing blood velocities. Thus the potential utility of these MB contrast agents, at least as suggested by this 1-D analysis, appears enhanced in the slower flow regimes. This may be relevant for improved visualization of smaller vessels with slower blood velocities with svOCT imaging.

The reasons for the observed decrease in decorrelation times may be twofold: (i) in the no-flow regime, larger particles (RBC) undergo slower Brownian motion than the scatterers in the blood $+\mathrm{MB}$ mixture, with the latter's smaller average diameter of $\sim 1.2 \mu \mathrm{m}$ increasing the rapidity of temporal variation and signal intensity fluctuations; (ii) in the flow regime, a given $\mathrm{MB}$ ensemble is continuously being replaced by another, resulting in a higher temporal variation of the scattered OCT signal and higher intensity fluctuations for a given applied velocity (flow rate). As seen from Fig. 3, the former effect is considerably stronger than the latter.

\subsection{Contrast Enhancement of Two-Dimensional Structural Images}

Structural OCT images, corresponding image contrasts, and ratios of blood $+\mathrm{MB}$ to blood contrasts acquired for different flow conditions are shown in Fig. 4. Although structural snapshot images show little visual evidence of MB contrast enhancement in Figs. 4(a) and 4(d), their averaged ROI intensities used for $C_{\mathrm{W}}$ calculation via Eq. (2) clearly demonstrate the contrast difference for both microtube diameters in Figs. 4(b) and 4(e). Dynamic OCT signal changes better reflect the contrast difference as shown in standard deviation image [Fig. 4(c)], calculated from eight subsequent structural images at $0.5 \mathrm{~mm} / \mathrm{s}$ blood flow velocity.

Similar to the temporal 1-D analysis (Fig. 3), the level of enhancement in Figs. 4(b) and 4(e) diminishes with increasing flow, but does not disappear (asymptote to unity) even for fast flows; as seen, there are still differences in the blue versus red histogram bars. Interestingly, despite the more than twofold difference in contrast values between 300- and 600- $\mu$ m diameter microtubes [note the different vertical scales in Figs. 4(b) and 4(e)], ratios of blood $+\mathrm{MB}$ to blood contrasts (height of red histogram bars divided by height of blue ones) for both of them are similar [Fig. 4(f)]. This likely stems from the fact that contrast in OCT structural images is based on the refractive index mismatches in the interrogated material. By introducing $\mathrm{MB}$ to the blood, the refractive index mismatches increase which increases scattering. This phenomenon is likely independent of the flow rate and yields additional structural image contrast, compared to blood-only conditions.

The two fold difference in contrast values between 300 - and 600- $\mu \mathrm{m}$ microtubes in Figs. 4(b) and 4(e) can be explained by the chosen contrast evaluation method. The OCT signal is lower
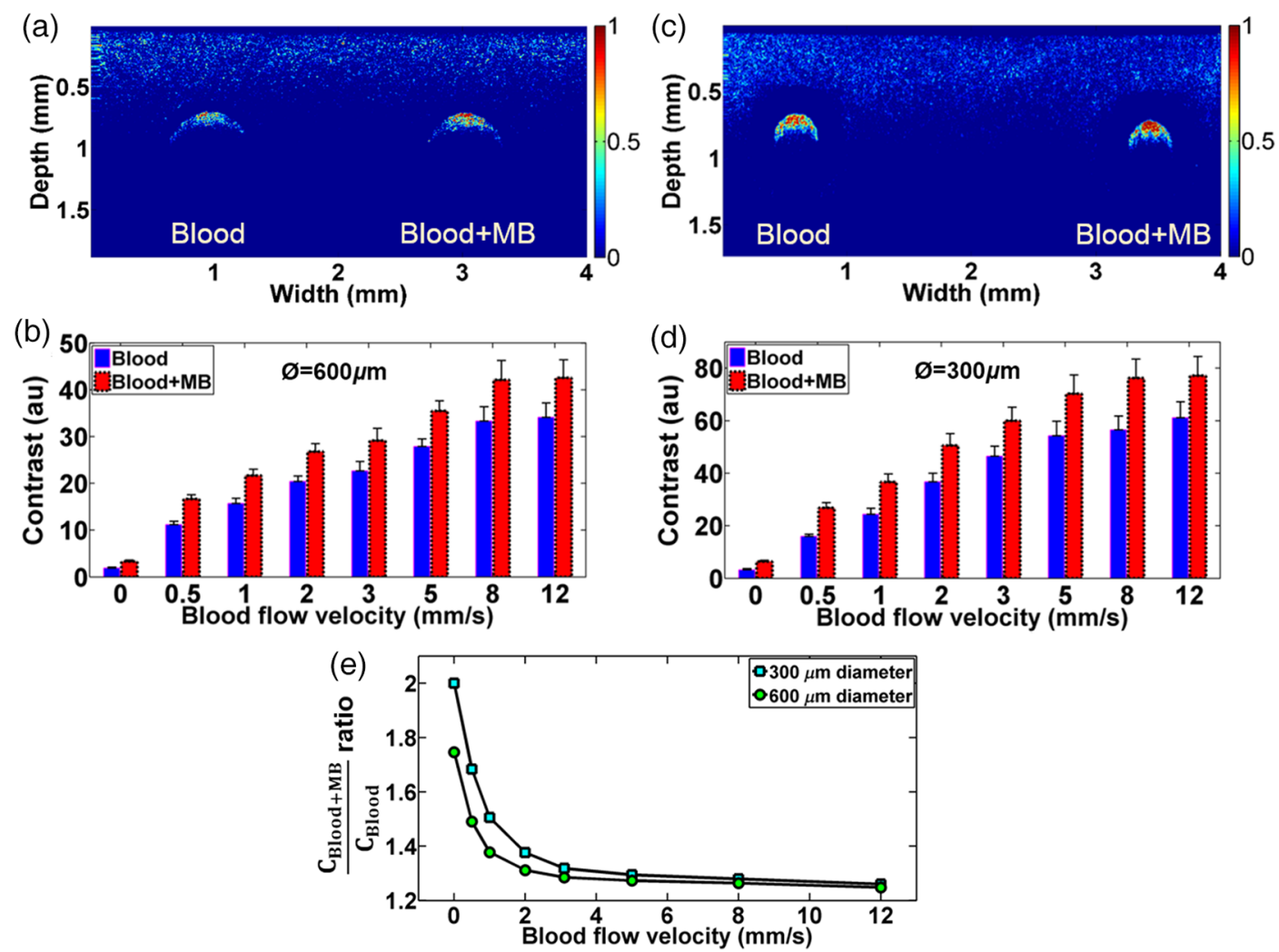

Fig. 5 (a) SVOCT image of the phantom with $600-\mu \mathrm{m}$ microtubes calculated from eight subsequent structural images at $0.5 \mathrm{~mm} / \mathrm{s}$ blood flow velocity; (b) svOCT image contrast for $600-\mu \mathrm{m}$ microtubes; (c) svOCT image of the phantom with $300-\mu \mathrm{m}$ microtubes at $0.5 \mathrm{~mm} / \mathrm{s}$ blood flow velocity; (d) svOCT image contrast for 300- $\mu \mathrm{m}$ microtubes. Error bars in (b) and (d) were evaluated from data at 200 different locations within each phantom. Note the different vertical scales in (b) and (d), see text for explanation; (e) ratio of blood $+\mathrm{MB} / \mathrm{blood}$ sv contrasts for both microtube diameters. 
at the bottom of the chosen half-circles of microtube lumens [see Figs. 2, 4(a), and 4(d)] for 600- $\mu \mathrm{m}$ diameter microtubes in comparison to $300-\mu \mathrm{m}$ diameter ones. As contrast evaluation is based on averaged ROI intensity, more low intensity pixels in the larger microtube's center will result in lower contrast value. The reason for the observed difference (especially at low flows) between contrast ratio curves in Fig. 4(f) may be in the faster RBC settling at low flows due to less intensive mixing/laminarity within the microtube of bigger diameter. We consider these effects not significant in the context of real in-vivo blood flow velocities reported in the literature for comparable vessel diameters $(\sim 8 \mathrm{~mm} / \mathrm{s}$ or faster). What is important here is a higher structural image contrast in the presence of MB in all experiments, more pronounced at low flows. Findings in Secs. 3.1 and 3.2 now set the stage for dynamic OCT image contrast investigation, as per sv studies discussed as follows.

\subsection{Contrast Enhancement of Two-Dimensional and Three-Dimensional Speckle Variance Optical Coherence Tomography Images}

As pointed out in Sec. 2, in the svOCT mode, the B-scan acquisition time was set to $25 \mathrm{~ms}$ between the $N=8$ frames. This was (1) fast enough that signals from stationary agar did not decorrelate between frames for svOCT signal to approach zero, and (2) sufficiently slow to ensure complete interframe decorrelation (high svOCT signal) for pixels representing blood (see Fig. 3 for the decorrelation time plots).
Figures 5(a) and 5(c) show the svOCT images corresponding to the structural images in Figs. 4(a) and 4(d) at $0.5 \mathrm{~mm} / \mathrm{s}$ blood flow velocity. As seen, there are more bright pixels in the lumen area of $300-\mu \mathrm{m}$ diameter microtubes in comparison to $600 \mu \mathrm{m}$ ones, likely due to OCT signal wash-out in bigger microtubes. Similar to structural contrast case, this also caused $\sim$ twofold difference between corresponding sv contrasts in the two microtubes [Figs. 5(b) and 5(d)]; note the different vertical scales.

Assessment of the ratio between blood $+\mathrm{MB}$ and blood contrasts [Fig. 5(e)] revealed a slightly higher contrast for smaller diameter microtube at low flows (similar trends to the structural contrast ratio metric above). Despite this similarity, it is important to emphasize that svOCT contrast values were found to be (i) 2 orders of magnitude higher than structural contrast values [see Figs. 4(b), 4(e), 5(b), and 5(d) for comparison], and (ii) 1.3 to 2 times higher for blood $+\mathrm{MB}$ mixture than for blood for flows up to $3 \mathrm{~mm} / \mathrm{s}$ [see Fig. 5(e)].

Moving on potential practical implementation of svOCT vascular contrast enhancement with MB added to the blood stream, we present en-face ( $x-y$ lateral dimensions) average intensity projections of volumetric phantom scans in Fig. 6. There are eight projections for different flow conditions of a phantom with embedded $600-\mu \mathrm{m}$ microtubes. The average velocities are indicated in each panel, and the flow direction is labeled with black arrows.

The average contrast values for these panels with deviations from phantom to phantom (error bars) can be found in Fig. 5(b).
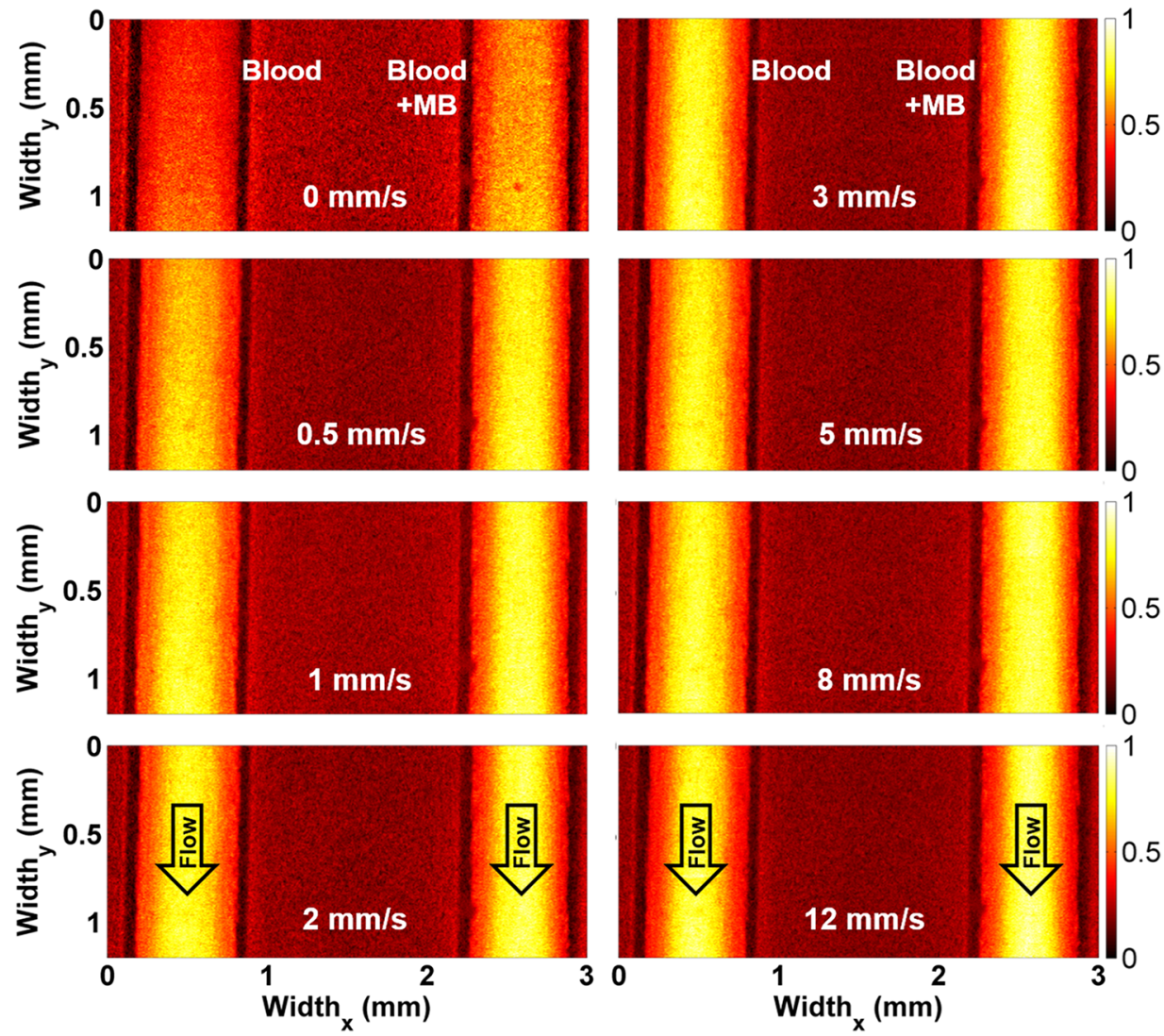

Fig. 6 En-face $(x-y$ lateral dimensions) svOCT average intensity projections of volumetric phantom scans. Eight projections corresponding to eight flow conditions in $600-\mu \mathrm{m}$ diameter microtubes with blood (left microtube) and blood + MB mixture (right microtube). Image intensities are normalized to the maximum value. The flow direction is labeled with black arrows. The OCT slow scanning axis is antiparallel to the flow direction. 
It is noticeable that at 0 to $1 \mathrm{~mm} / \mathrm{s}$ flows there is a slight gradient of svOCT signal in $Y$-direction along microtubes. That is because svOCT imaging takes time (tens of seconds for each panel) and images reflect RBC gradual settlement at low flows. As other parameters (blood flow, MB concentration, hematocrit) remain unchanged, from these low flow images, where brighter svOCT signal is seen at right microtubes (filled with blood + MB mixture), we can assume that gradual RBC settlement occurs slower than in left microtubes (filled with blood). The possible reason for that may be $\mathrm{MB}$ particles exhibiting faster Brownian motion relative to $\mathrm{RBC}$ at low flows due to their smaller size (as mentioned in Sec. 3.1). One of the recent reports ${ }^{29}$ on OCT study of diffusive versus flow contributions to backscattered OCT signal at low flows for small particle sizes supports this hypothesis. As a result, faster motion of surrounding smaller particles contributes to slower RBC settlement, leading to more rapid intensity fluctuations and higher svOCT contrast.

However, at higher velocities the flow contribution to OCT scattering dominated over diffusive (Brownian motion) effects. ${ }^{29}$ In our experiments, this leads to increased role of $\mathrm{RBC}$, dominating over MB contribution and therefore diminishing the difference between blood and blood $+\mathrm{MB}$ mixture contrasts in both snapshot structural OCT and dynamic svOCT images. Indeed, the common trends observed here from different analysis methods [Figs. 3, 4(b), 4(e), 5(b), and 5(d), as well as faster flow panels in Fig. 6] support these suggested mechanisms.

For potential in-vivo applications of MB-enhanced OCT, assessment of microvascular response to treatment (ultrasound ablation, photodynamic, chemical and radiation therapies of tissues) appears promising. For example, in the last scenario, ${ }^{30}$ smallest microvessels with low blood flow are known to be most sensitive to irradiation; however, their detection with svOCT is difficult due to low SNR especially at lower depths. Adding MB to the blood stream before OCT imaging may increase SNR for these vessels and improve their detection and thus help visualize their response to irradiation. Further, vascular-targeted treatments (e.g., angiogenic or antiangiogenic therapies) generally affect the vascular functionality. Preimaging the vasculature, then adding $\mathrm{MB}$ and imaging vasculature again may reveal "compromised" stagnant/blocked vessels with unchanged contrast (no MB) versus "functioning" increasedcontrast vessels (with $\mathrm{MB}$ ).

\section{Conclusions}

We have demonstrated that the addition of MBs to blood improves the OCT structural and sv contrast at different flow conditions in two differently sized model vessels. Various OCT measurement modes were employed to demonstrate these effects, which decreased overall with increasing blood flow rates. This is potentially significant for in vivo deployment, perhaps leading to MB-enabled detection of the finest microvascular features in tissues (capillaries). These smallest microvessels are difficult to detect otherwise, yet are important of early pathology detection and treatment response monitoring. ${ }^{31}$

Further, as MB-based OCT contrast appears to decrease with flow velocity, this methodology may potentially help visualize stationary (no blood flow) conditions as blocked/stagnant microvessels amongst the flowing microvascular network, thus isolating dysfunctional perfusion foci that are otherwise difficult to detect. These findings warrant further investigations in utilizing MB for tissue hemodynamic investigations and for microvasculature contrast enhancement in OCT angiography.

\section{Acknowledgments}

This study was supported by the Canadian Institutes of Health Research, Ryerson University Health Research Grant. V.D. was also supported by Alexander Graham Bell Canada Graduate Scholarship from the Natural Sciences and Engineering Research Council of Canada.

\section{References}

1. A. K. Dunn et al., "Introduction: feature issue on in vivo microcirculation imaging," Biomed. Opt. Express 2(7), 1861-1863 (2011).

2. E. V. Zagaynova et al., "Contrasting properties of gold nanoparticles for optical coherence tomography: phantom, in vivo studies and Monte Carlo simulation," Phys. Med. Biol. 53(18), 4995-5009 (2008).

3. T. S. Troutman, J. K. Barton, and M. Romanowski, "Optical coherence tomography with plasmon resonant nanorods of gold," Opt. Lett. 32(11), 1438-1440 (2007).

4. T. M. Lee et al., "Engineered microsphere contrast agents for optical coherence tomography," Opt. Lett. 28(17), 1546-1548 (2003).

5. J. C. Y. Kah et al., "Control of optical contrast using gold nanoshells for optical coherence tomography imaging of mouse xenograft tumor model in vivo," J. Biomed. Opt. 14(5), 054015 (2009).

6. C. S. Kim et al., "Enhanced detection of early-stage oral cancer in vivo by optical coherence tomography using multimodal delivery of gold nanoparticles," J. Biomed. Opt. 14(3), 034008 (2009).

7. C. Yang, "Molecular contrast optical coherence tomography: a review," Photochem. Photobiol. 81(2), 215-237 (2005).

8. K. M. Au et al., "Anti-biofouling conducting polymer nanoparticles as a label-free optical contrast agent for high resolution subsurface biomedical imaging," Biomaterials 34(35), 8925-8940 (2013).

9. M. J. Hsu et al., "Characterization of individual ultrasound microbubble dynamics with a light-scattering system," J. Biomed. Opt. 16(6), 067002 (2011).

10. J. K. Barton, J. B. Hoying, and C. J. Sullivan, "Use of microbubbles as an optical coherence tomography contrast agent," Acad. Radiol. 9(1), S52-S55 (2002).

11. H. Assadi, R. Karshafian, and A. Douplik, "Optical scattering properties of intralipid phantom in presence of encapsulated microbubbles," Int. J. Photoenergy 2014, 1-9 (2014).

12. M. A. Borden et al., "Ultrasound radiation force modulates ligand availability on targeted contrast agents," Mol. Imaging 5(3), 139-147 (2006).

13. M. Todorova et al., "Antitumor effects of combining metronomic chemotherapy with the antivascular action of ultrasound stimulated microbubbles," Int. J. Cancer 132(12), 2956-2966 (2013).

14. D. E. Goertz et al., "Antitumor effects of combining docetaxel (taxotere) with the antivascular action of ultrasound stimulated microbubbles," PLoS One 7(12), e52307 (2012).

15. G. J. Czarnota et al., "Tumor radiation response enhancement by acoustical stimulation of the vasculature," Proc. Natl. Acad. Sci. U. S. A. 109(30), E2033-E2041 (2012).

16. C. Tarapacki and R. Karshafian, "Enhancing laser therapy using PEGylated gold nanoparticles combined with ultrasound and microbubbles," Ultrasonics 57, 36-43 (2015).

17. A. Mariampillai et al., "Speckle variance detection of microvasculature using swept-source optical coherence tomography," Opt. Lett. 33(13), 1530-1532 (2008)

18. M. S. Mahmud et al., "Review of speckle and phase variance optical coherence tomography to visualize microvascular networks," $J$. Biomed. Opt. 18(5), 050901 (2013).

19. I. Gorczynska et al., "Comparison of amplitude-decorrelation, specklevariance and phase-variance OCT angiography methods for imaging the human retina and choroid," Biomed. Opt. Express 7(3), 911 (2016).

20. L. A. Matveev et al., "Hybrid M-mode-like OCT imaging of threedimensional microvasculature in vivo using reference-free processing of complex valued B-scans," Opt. Lett. 40(7), 1472-1475 (2015). 
21. B. J. Vakoc et al., "Cancer imaging by optical coherence tomography: preclinical progress and clinical potential," Nat. Rev. Cancer 12(5), 363-368 (2012).

22. H. J. van Staveren et al., "Light scattering in intralipid- $10 \%$ in the wavelength range of 400-1100 nm," Appl. Opt. 30(31), 4507-4514 (1991).

23. P. Di Ninni, F. Martelli, and G. Zaccanti, "Intralipid: towards a diffusive reference standard for optical tissue phantoms," Phys. Med. Biol. 56(2), N21-N28 (2011).

24. B. Fagrell, A. Fronek, and M. Intaglietta, "A microscope-television system for studying flow velocity in human skin capillaries," Am. J. Physiol. Heart Circ. Physiol. 233(2), H318-H321 (1977).

25. P. Bagchi, "Mesoscale simulation of blood flow in small vessels," Biophys. J. 92(6), 1858-1877 (2007).

26. H. N. Mayrovitz, D. Larnard, and G. Duda, "Blood velocity measurement in human conjunctival vessels," Cardiovasc. Dis. 8(4), 509-526 (1981).

27. T. A. Fritz and E. C. Unger, "Phase I clinical trials of MRX-115. A new ultrasound contrast agent," Invest. Radiol. 32(12), 735-740 (1998).

28. A. Mariampillai et al., "Optimized speckle variance OCT imaging of microvasculature," Opt. Lett. 35(8), 1257-1259 (2010).

29. N. Weiss, T. G. van Leeuwen, and J. Kalkman, "Simultaneous and localized measurement of diffusion and flow using optical coherence tomography," Opt. Express 23(3), 3448-3459 (2015).

30. A. Maeda et al., "In-vivo optical imaging of tumor and microvascular response to ionizing radiation," PLoS One 7(8), e42133 (2012).

31. M.-T. Tsai et al., "Investigation of temporal vascular effects induced by focused ultrasound treatment with speckle-variance optical coherence tomography," Biomed. Opt. Express 5(7), 2009-2022 (2014).

Homa Assadi is a biomedical physics $\mathrm{PhD}$ candidate at the Ryerson University, Canada. She received her BS and MS degrees in physics from the University of Isfahan and Azad University, respectively. Her current research interests in optical devices such as spectroscopy, diffuse reflectance imaging, OCT imaging, LS imaging, and microbubbles contrast agents. She is a member of OSA and SPIE.

Valentin Demidov is a medical biophysics $\mathrm{PhD}$ candidate at University of Toronto, Canada. His research is focused on development and application of new diagnostic techniques based on the use of optical coherence tomography (OCT). Current initiatives include volumetric optical microangiography, quantification of microvascular changes in tissues with biologically relevant metrics, and alternative contrast mechanisms in OCT (image texture analysis, speckle temporal synchronization effects, interstitial fluids mapping in healthy and diseased tissues).

Raffi Karshafian: Biography is not available.

Alexandre Douplik is an associate professor of the Department of Physics at Ryerson University, Toronto, since August 2011, and also residential scientist of Keenan Research Centre at St. Michael Hospital, Toronto (since 2015). In 2007 to 2011, he was a guest professor of Nuremberg-Erlangen University, Germany. Before 2007, he was a scientific associate at Biophysics/Bioimaging Division in Ontario Cancer Institute in Toronto (2006 to 2007) and senior researcher of Xillix Technologies Corporation, Vancouver (2002 to 2006).

I. Alex Vitkin is a professor of Medical Biophysics and Radiation Oncology at the University of Toronto, a senior scientist at the University Health Network, and a clinical physicist at Princess Margaret Cancer Centre (all in Toronto, Canada). He has published $\sim 170$ papers + book chapters on biophotonics, primarily on tissue polarimetry and optical coherence tomography. He is also a fellow of OSA and SPIE, and a professor at the Nizhny Novgorod State Medical Academy (Russia). 